九州大学学術情報リポジトリ

Kyushu University Institutional Repository

\title{
In situ third-order interferometric autocorrelation of a femtosecond deep- ultraviolet pulse
}

\section{Miyoshi, Yuki}

Department of Applied Chemistry, Graduate School of Engineering, Kyushu University

Zaitsu, Shinichi

Department of Applied Chemistry, Graduate School of Engineering, Kyushu University

Imasaka, Totaro

Division of Translational Research, Center for Future Chemistry, Kyushu University I

Department of Applied Chemistry, Graduate School of Engineering, Kyushu University

http://hdl. handle. net/2324/25514

出版情報：Applied Physics B : Lasers and Optics. 103 (4), pp.789-794, 2011-06-01. SpringerVerlag

バージョン :

権利関係: (C) Springer-Verlag 2010 


\title{
In-situ third-order interferometric
}

\section{autocorrelation of a femtosecond}

\section{deep-ultraviolet pulse}

\author{
Y. Miyoshi ${ }^{1}$, S. Zaitsu ${ }^{1}$, T. Imasaka ${ }^{1,2}$ \\ 1 Department of Applied Chemistry, Graduate School of Engineering, Kyushu \\ University, 744 Motooka, Nishi-ku, Fukuoka 819-0395, Japan \\ ${ }^{2}$ Division of Translational Research, Center for Future Chemistry, Kyushu Uni- \\ versity, 744 Motooka, Nishi-ku, Fukuoka 819-0395, Japan
}

Received: date / Revised version: date

\begin{abstract}
The interferometric measurement of an ultrashort optical pulse is demonstrated using three-photon ionization of Xe in a time-of-flight mass spectrometer (MS). This approach allowed measurement of the third-order fringe-resolved autocorrelation trace of an ultrashort optical pulse in the deep-ultraviolet (DUV) region. The pulse can be measured in-situ using the same MS that is employed in ultrafast spectroscopy. Sensitivity $\left(>3 \times 10^{7}\right.$ W) was sufficient to measure a DUV pulse used in such applications. This setup has the potential to measure the temporal duration of a broadband
\end{abstract} Send offprint requests to: imasaka@cstf.kyushu-u.ac.jp 
(204 - $306 \mathrm{~nm})$ ultrashort optical pulse with no additional distortion in the temporal characteristics.

\section{Introduction}

Evolution in the research of ultrafast science has increased the demand for new technology that can simply and accurately determine the temporal characteristics of an ultrashort optical pulse for frequencies that range from the near-infrared (NIR) to the deep-ultraviolet (DUV). In particular, the measurement of an optical pulse consisting of only a few cycles of electromagnetic wave, and having an extremely broad bandwidth in the frequency domain, presents serious difficulties. Problems include separation of the measurement and application locations. Ultrashort optical pulses with a broad frequency range are significantly affected by propagation of the beam through a variety of media, even air. Complications arise when the waveform of a pulse must be accurately determined at a point where the pulse is actually utilized to probe the ultrafast behavior of matter. To solve this problem, mass spectrometers (MSs) have been widely used to measure the temporal nature of ultrashort optical pulses [1-7]. MSs are currently employed as basic tools in research on the ultrafast phenomena of atoms and molecules $[8,9]$, atto-second time-scale science $[10,11]$, and trace analysis $[12,13]$. An ultrashort optical pulse can be manipulated in a MS with no change in its temporal characteristics because the chamber is evacuated, so the pulse is not distorted. Therefore, using the same MS, the temporal characteristics of 
ultrashort optical pulses can be measured, while simultaneously exploring ultrafast phenomena.

A MS is most effective for measuring ultrashort optical pulses in the wavelength region shorter than visible, where the dispersion due to the medium is more significant than in the visible and NIR regions. This technique is based on the multi-photon ionization (MPI) of a gaseous medium having no absorption band in the ultraviolet (UV) region [14-17]. The $n$ photon ionization generally yields a nonlinear signal proportional to the $n$-th order of the input pulse intensity. This provides a nonlinear response, which is necessary for obtaining an autocorrelation trace to measure the pulse duration. Moreover, the use of MPI in the measurement of an UV ultrashort optical pulse can solve the problem arising from a lack of nonlinear crystalline materials available in this spectral region. The small frequency dependence of non-resonant ionization in a wide spectral range does little to limit phase-matching and offers the possibility of measuring the temporal profile of an extremely short optical pulse.

Herein is a description of the fringe-resolved autocorrelation (FRAC) measurement of an ultrashort optical pulse in the DUV region based on the three-photon ionization of Xe using a time-of-flight mass spectrometer (TOF/MS). Since Xe acts as a nonlinear material having an ionization potential of $12.1 \mathrm{eV}$, it can be used to obtain a third-order nonlinear signal ranging from $204 \mathrm{~nm}(6.1 \mathrm{eV})$ to $306 \mathrm{~nm}(4.0 \mathrm{eV})$. The wide wavelength range for a nonlinear response of Xe offers the potential for the measurement 
of extremely short DUV pulses; the minimum measurable pulse duration expected from this measurable spectral range is calculated to be $1.9 \mathrm{fs}$, which assumes the spectrum has a rectangular shape. Section 2 summarizes the expected shape of the third-order FRAC trace and the parameters necessary to evaluate the pulse duration. After describing the experimental setup in Section 3, the measured FRAC trace is reported in Section 4 to determine the temporal profile of the Fourier-transform-limited (FTL) pulse in the DUV region $(262 \mathrm{~nm})$. The nonlinear dependence of the Xe ionization signal on the input pulse intensity and the applicability of Xe to a low-power or chirped pulse are also discussed in Section 4. Finally, the experimental results are summarized and the work is concluded in Section 5 .

\section{Nonlinear Autocorrelation Trace}

Nonlinear autocorrelation for measuring the temporal profile of an ultrashort optical pulse is divided into two major categories: fringe-resolved (interferometric) autocorrelation (FRAC) and intensity (non-interferometric) autocorrelation (IAC). The AC traces obtained by these methods can be expressed by the following equations:

$$
\begin{gathered}
I_{\text {frac }}^{n}(\tau)=\int_{-\infty}^{\infty}\left|(E(t)+E(t-\tau))^{n}\right|^{2} d t, \\
I_{i a c}^{n}(\tau)=\int_{-\infty}^{\infty}\left(I(t)^{n-1} I(t-\tau)\right) d t,
\end{gathered}
$$

where $E(t)$ and $I(t)$ are the amplitude of the electromagnetic field and the intensity of the measured optical pulse as a function of time, respectively. $\tau$ 
is the relative delay between identical pulses, and $n$ is the order of nonlinearity in response to the input pulse. Since the second-order susceptibility, $\chi^{(2)}$, works efficiently in the nonlinear process under phase-matched conditions, the second-order autocorrelation trace $(n=2)$ can be widely used to evaluate the temporal profile of an ultrashort optical pulse. Although the third-order autocorrelation has a lower sensitivity because the third-order susceptibility, $\chi^{(3)}$, is small, there are several potential advantages: (1) elimination of ambiguity in the time domain, (2) availability of materials used in the UV region, and (3) a broad frequency domain for measurement. These preferable features have been utilized in the measurement of ultrashort optical pulses using $\chi^{(3)}$ based on polarization-gating [18], self-diffraction [20, 21], degenerate four-wave mixing [19], and third harmonic generation [22, 23].

With the AC measurement approach, the envelope of an ultrashort optical pulse is usually calculated by assuming it is a Gaussian function $\left(\exp ^{-t^{2} / 2}\right)$, which is widely used as a typical optical pulse shape, or a hyperbolic secant function $(\operatorname{sech}(t))$, which is mainly used for mode-locked laser pulses. For these two types of envelope shapes, the ratios of the fullwidth at half maximum (FWHM), $\Delta \tau$, of the $\mathrm{AC}$ trace to that in real time, $t_{p}$, are calculated using Equations (1) and (2) for $n=2$ and 3 and are summarized in Table 1. Although these values are strictly valid only for an FTL pulse, they can be used as a good approximation for chirped pulses in a certain range of pulse duration. Figure 1 shows typical third-order FRAC 
traces for a Gaussian-shaped FTL pulse (Fig. 1(a)), as well as that of a chirped pulse (Fig. 1(b)). The intensity ratio of the peak $(\tau=0)$ to the baseline $(\tau=\infty)$ is 32 , whereas it is 8 for a second-order AC trace. Thus, the experimentally obtained ratio can be used as a criterion to validate the measurement. In Fig. 1(b), a chirped pulse has a shape that includes an undulated baseline at the wings of the FRAC trace. In this case, the pulse duration can be estimated from the profile of the IAC trace extracted by filtering the high frequency components from the measured FRAC trace.

\section{Experimental setup}

The experimental setup used in the present work is shown in Fig. 2. An NIR ultrashort optical pulse from a Ti:sapphire regenerative amplifier (Thales, $784 \mathrm{~nm}, 100 \mathrm{fs}, 1 \mathrm{~mJ}, \Delta \lambda=9 \mathrm{~nm}, 1 \mathrm{kHz}$ ) was focused into a nonlinear optical crystal $\left(\mathrm{KD}^{*} \mathrm{P}\right)$ to generate a near-ultraviolet (NUV) second harmonic (392 $\mathrm{nm}$ ) emission. The third harmonic (TH) emission (262 nm, $\Delta \lambda=1 \mathrm{~nm}$, $100 \mu \mathrm{J}$ ) was subsequently generated by sum frequency mixing of the NIR and NUV pulses in another KD*P crystal. The DUV beam was passed through a pulse compressor consisting of a pair of silica prisms, one of which was translated to control the pulse duration. The beam was divided into two parts using a beam splitter in a Michelson interferometer. The divided beams were recombined collinearly after adding a time delay to one of them. The recombined beam was focused into a laboratory-made TOF/MS [24] through a silica window using a lens with a 300-mm focal length. Nitrogen 
monoxide (NO) and xenon (Xe), contained in a reservoir made of an inert plastic bag, were continuously injected through a silica capillary into the TOF/MS at the position where the laser beam was focused. The ions formed by multi-photon ionization were detected with an assembly of microchannel plates after drifting in the TOF tube. The intensity of the ion signal was displayed as a function of the flight time using an oscilloscope (Tektronix, TDS5104) to construct a mass spectrum. The signal intensities of the peaks corresponding to the molecular ions of NO or Xe were measured using a boxcar integrator. The averaged signal was monitored while introducing a delay between the two identical pulses and using an oscilloscope to record the $\mathrm{AC}$ trace.

\section{Results and Discussions}

Figure 3(a) shows a mass spectrum recorded using Xe as a medium for photo-ionization. The mass spectrum consists of seven peaks. The intensity distribution of these peaks was in reasonable agreement with the natural abundance of Xe isotopes: $2 \%\left({ }^{128} \mathrm{Xe}\right), 26 \%\left({ }^{129} \mathrm{Xe}\right), 4 \%\left({ }^{130} \mathrm{Xe}\right), 21 \%$ $\left({ }^{131} \mathrm{Xe}\right), 27 \%\left({ }^{132} \mathrm{Xe}\right), 10 \%\left({ }^{134} \mathrm{Xe}\right)$, and $9 \%\left({ }^{136} \mathrm{Xe}\right)[25]$. The mass resolving power, which is defined by $m / \Delta m$, was $c a .600$ for the largest isotope peak $\left({ }^{132} \mathrm{Xe}\right)$. For FRAC measurement of the temporal profile, the output signal of the detector should be nonlinearly dependent on the input pulse energy. To examine this signal nonlinearity, two different gate widths with a boxcar

integrator were used: one to monitor a single peak of ${ }^{129} \mathrm{Xe}$ and the other to 
monitor the peaks due to all the isotopes. The signal was recorded for several pulse energies that were changed using a variable neutral-density filter. A double logarithmic plot of the data is shown in Fig. 3(b). The slopes of the fitted lines for ${ }^{129} \mathrm{Xe}(2 \mu \mathrm{J}-17 \mu \mathrm{J})$ and all the isotopes, $(3 \mu \mathrm{J}-16 \mu \mathrm{J})$, were both 3.0, suggesting that Xe isotopes were ionized through a non-resonant three-photon ionization process induced by an ultrashort DUV pulse above the input energy of $3 \mu \mathrm{J}$. This can be attributed to the fact that the photon energy of the DUV pulse ( $E=4.7 \mathrm{eV}$, i.e. $262 \mathrm{~nm})$ is less than one-half ( 6.06 $\mathrm{eV}$, i.e. $204 \mathrm{~nm})$ and more than one-third $(4.04 \mathrm{eV}$, i.e. $306 \mathrm{~nm})$ of the ionization energy for Xe $\left(E_{i}=12.1 \mathrm{eV}\right)$, i.e., $2 E<E_{i}<3 E$. The multi-photon ionization spectrum reported in previous work [26] showed two prominent signals at $383-388 \mathrm{~nm}$ and $437-441 \mathrm{~nm}$, which were caused by threephoton resonance via the $5 d$ level and four-photon resonance via the $4 f$ level, respectively. This implies that Xe isotopes can provide a third-order nonlinear response over a wide spectral range $(204-306 \mathrm{~nm})$ and would be useful for measuring the temporal profiles of ultrashort optical pulses by avoiding these two resonance wavelengths in the DUV region. The secondorder nonlinear response measured using $\mathrm{NO}$ as a nonlinear medium for multiphoton ionization has already been reported elsewhere [16]. An ionization energy that is lower for $\mathrm{NO}(9.26 \mathrm{eV})$ than for $\mathrm{Xe}$, and the high energy level of the first excited singlet state $(4.13 \mathrm{eV})$, limits the bandwidth for a second-order nonlinear response. Moreover, the energy levels caused by the molecular motions of NO deteriorate the shape of the FRAC trace. The 
broad bandwidth for nonlinear response and the simple structure of energy levels will be significant advantages of the use of Xe, instead of NO, as a nonlinear material for the characterization of ultrashort optical pulses using multiphoton ionization.

In the following FRAC measurement, NO [16] and Xe were simultaneously introduced into the TOF/MS and the ion signals from both were recorded in a single mass spectrum. The simultaneous introduction of Xe and NO into a MS enabled the direct comparison of two different FRAC traces from the peak signals with different input-energy dependencies. First, the position of the prisms in the pulse compressor were set by observing the second-order FRAC trace using the ion signal of NO to minimize the temporal duration of the DUV pulse, i.e., the FTL pulse. Then, a FRAC trace was recorded using the signal obtained by integrating all the Xe isotope peaks. Figures 4(a) and (b) show the FRAC traces arising from Xe and NO signals, respectively. The FRAC trace shown in Fig. 4(a) is consistent with the theoretically predicted shape of the FTL pulse shown in Fig. 1(a); the ratio of the intensities of the peak and baseline was 32. An interferometric fringe pattern is also shown in the inset of Fig. 4(a). The visible fringe is clear, and the average time period of one cycle was used for the calibration of the time delay in the FRAC trace. The FWHM of the FRAC trace $(\Delta \tau)$ divided by the theoretically predicted ratio $(\Delta \tau / \Delta t=1.36)$ for a sech ${ }^{2}$ pulse yielded an estimated pulse duration $(\Delta t)$ of $101 \mathrm{fs}$. The pulse width calculated from the FWHM of the NO-based FRAC trace (Fig. 4(b)) was $101 \mathrm{fs,} \mathrm{which} \mathrm{was}$ 
in excellent agreement with the value obtained from the Xe-based FRAC trace. This result clearly suggests that the third-order FRAC trace obtained using Xe provides a useful means for measuring the temporal profile of an FTL DUV pulse in a similar manner to the second-order FRAC trace using NO. To the best of our knowledge, this is the first demonstration of a pulse duration measurement in the DUV region based on a third-order FRAC.

To determine the minimum input pulse energy for recording a thirdorder FRAC trace, the FRAC trace was measured while the energy of the DUV pulse was decreased using a neutral-density filter. The signal-to-noise ratio in the FRAC traces deteriorated as pulse energy decreased. Figure 5(a) shows a FRAC trace measured at a pulse energy of $1.2 \mu \mathrm{J}$, for which the calculation of a peak-to-baseline ratio of 32 is difficult. However, the FRAC measurement at $>3 \mu \mathrm{J}$ provided a shape corresponding to the theoreticallypredicted trace with a ratio of 32 , suggesting that the minimum intensity of the DUV pulse was $\sim 3 \times 10^{7} \mathrm{~W}$. This limitation likely arose from the shot noise of the signal when the number of generated ions was rather small. This also can be explained by the results shown in Fig. 3(b), in which a DUV pulse with an energy lower than $3 \mu \mathrm{J}$ provides a low ionization efficiency, leading to scatter from the third-order nonlinear response curve. To measure a chirped pulse with a peak intensity that is lower than an FTL pulse, the AC trace of a DUV pulse was measured after adding a positive dispersion by increasing the path length in one of the prisms in the pulse compressor. The obtained FRAC trace is shown in Fig. 5(b). The curve (white line) is the 
IAC trace calculated by passing the FRAC trace through a low-pass filter. The IAC in Fig. 5(b) provided an increased pulse duration of 110 fs that was calculated using the ratio, $\Delta \tau / \Delta t=1.29$, by assuming a $\operatorname{sech}^{2}$ pulse shape. This value was apparently larger than that expected for an FTL pulse (101 fs). It should be noted that an undulation was observed in the baseline of the FRAC trace, which does not appear in the trace in Fig. 4(a) but does appear in the trace calculated for a chirped pulse (see Fig. 1(b)). This undulation can be attributed to frequency chirping (a time dependent frequency variation). Thus, this information can be used to evaluate the chirp in the DUV pulse. This type of interferometric measurement is useful for obtaining an FTL pulse in the process of dispersion compensation.

\section{Conclusion}

The present study demonstrates a novel approach for measuring the temporal characteristics of an ultrashort optical pulse in the DUV region, based on the three-photon ionization of Xe in a MS. This approach enabled measurement of the pulse width of a DUV pulse and the use of a MS in a variety of applications with no modification of the temporal characteristics of the pulse. The third-order FRAC trace of a DUV ultrashort optical pulse was successfully measured and this approach provided a simple and versatile method for measuring the temporal profile and differentiating an FTL pulse from a chirped one. This three-photon ionization scheme has sufficient sensitivity to measure a DUV ultrashort optical pulse, which is employed in 
spectroscopic research at a level of several $\mu \mathrm{J}$ and in trace analysis at $\sim 100$ $\mu J$. In addition, the pulse can be measured in-situ in the same physical location as the experimentation, which allows the use of extremely ultrashort optical pulses with no distortion of the temporal characteristics. Thus, this technique has the potential to be used in a wide range of research fields based on TOF/MS coupled with an ultrashort optical pulse in the DUV region.

Acknowledgements This research was partially supported by Grants-in-Aid for Scientific Research and the Global COE Program "Science for Future Molecular Systems" from the Ministry of Education, Culture, Sports, Science and Technology of Japan.

\section{References}

1. P. Tzallas, D. Charalambidis, N. A. Papadogiannis, K. Witte, G. D. Tsakiris, Nature 426, 267 (2003)

2. T. Sekikawa, T. Kanai, S. Watanabe, Phys. Rev. Lett. 91, 103902 (2003)

3. T. Sekikawa, A. Kosuge, T. Kanai, S. Watanabe, Nature 432, 605 (2004)

4. Y. Mairesse, O. Gobert, P. Breger, H. Merdji, P. Meynadier, P. Monchicourt, M. Perdrix, P. Salières, B Carré, Phys. Rev. Lett. 94, 173903 (2005)

5. Y. Nabekawa, T. Shimizu, T. Okino, K. Furusawa, H. Hasegawa, K. Yamanouchi, K. Midorikawa, Phys. Rev. Lett. 97, 153904 (2006)

6. O. Faucher, P. Tzallas, E.P. Benis, J. Kruse, A. P. Conde, C. Kalpouzos, D.

Charalambidis, Appl. Phys. B. 97, 505 (2009) 
7. F. Reiter, U. Graf, M. Schultze W. Schweinberger, H. Schröder, N. Karpowicz, A. M. Azzeer, R. Kienberger, F. Krausz, and E. Goulielmakis, Opt. Lett. 35. $2248(2010)$

8. A. H. Zewail, J. Phys. Chem. A 104, 5660 (2000)

9. I. V. Hertel, W. Radloff, Rep. Prog. Phys. 69, 1897 (2006)

10. F. Krausz, M. Ivanov, Rev. Mod. Phys. 81, 163 (2009)

11. P. B. Corkum, F. Krausz, Nature Phy.3, 381 (2007)

12. T. Imasaka, Chem. Rec. 8, 23 (2008)

13. C. Mullen, M. J. Coggiola, H. Oser, J. Am. Soc. Mass Spectrom. 20, 419 (2009)

14. D. M. Rayner, P. A. Hackett, C. Willis, Rev. Sci. Instrum. 53, 537 (1982)

15. Y. Kobayashi, T. Sekikawa, Y. Nabekawa, S. Watanabe, Opt. Lett. 23, 64 (1998)

16. S. Zaitsu, Y. Miyoshi, F. Kira, S. Yamaguchi, T. Uchimura, T. Imasaka, Opt. Lett. 32, 1716 (2007)

17. A. P. Conde, J. Kruse, O. Faucher, P. Tzallas, E. P. Benis, D. Charalambidis, Phys. Rev. A 79, 061405 (2009)

18. J. Etchepare, G. Grillon, A. Orszag, IEEE J. Quantum Electron. QE-19, 775 (1983)

19. H. Schulz, H. Schuler, T. Engers, D. von der Linde, IEEE J. Quantum Electron. QE-25, 2580 (1989)

20. W.L. Nighan Jr., T. Gong, L. Liou, P.M. Fauchet, Opt. Comm. 69, 339 (1989)

21. S. Backus, J. Peatross, Z. Zeek, A. Rundquist, G. Taft, M. M. Murnane, H.

C. Kapteyn, Opt. Lett. 21, 665 (1996)

22. D. Meshulach, Y. Barad, Y. Silberberg, J. Opt. Soc. Am. B 14, 2122 (1997) 
23. J. A. Squier, D. N. Fittinghoff, C. P. J. Barty, K. R. Wilson, M. Müller, G.

J. Brakenhoff, Opt. Comm. 147, 153 (1998)

24. T. Onoda, G. Saito, T. Imasaka, Anal. Chem. Acta 412, 213 (2000)

25. J. R. de Laeter, J. K. Böhlke, P. De Biévre, H. Hidaka, H. S. Peiser, K. J. R.

Rosman, P. D. P. Taylor, Pure Appl. Chem. 75, 683 (2003)

26. K. Aron, P. M. Johnson, J. Chem. Phys. 67, 5099 (1977) 

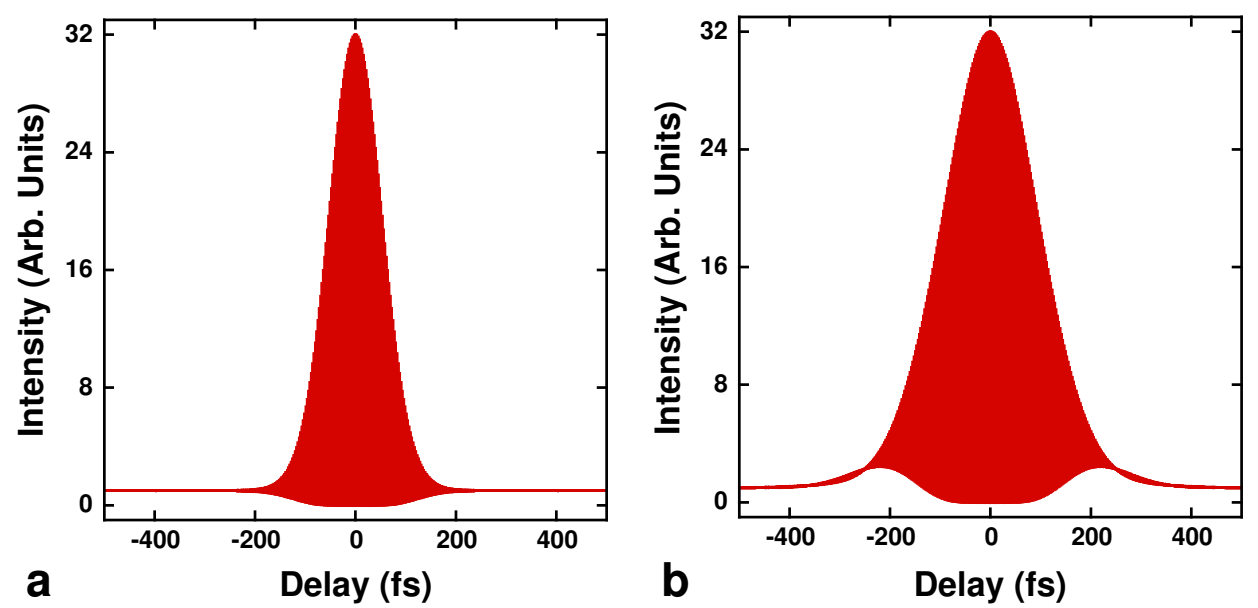

Fig. 1 The fringe-resolved autocorrelation trace calculated for ultrashort optical pulse with a $\operatorname{sech}^{2}$ shape. (a) FTL pulse with a duration of 100 fs. (b) Chirped pulse temporally extended by an additional group delay dispersion from the FTL pulse. 


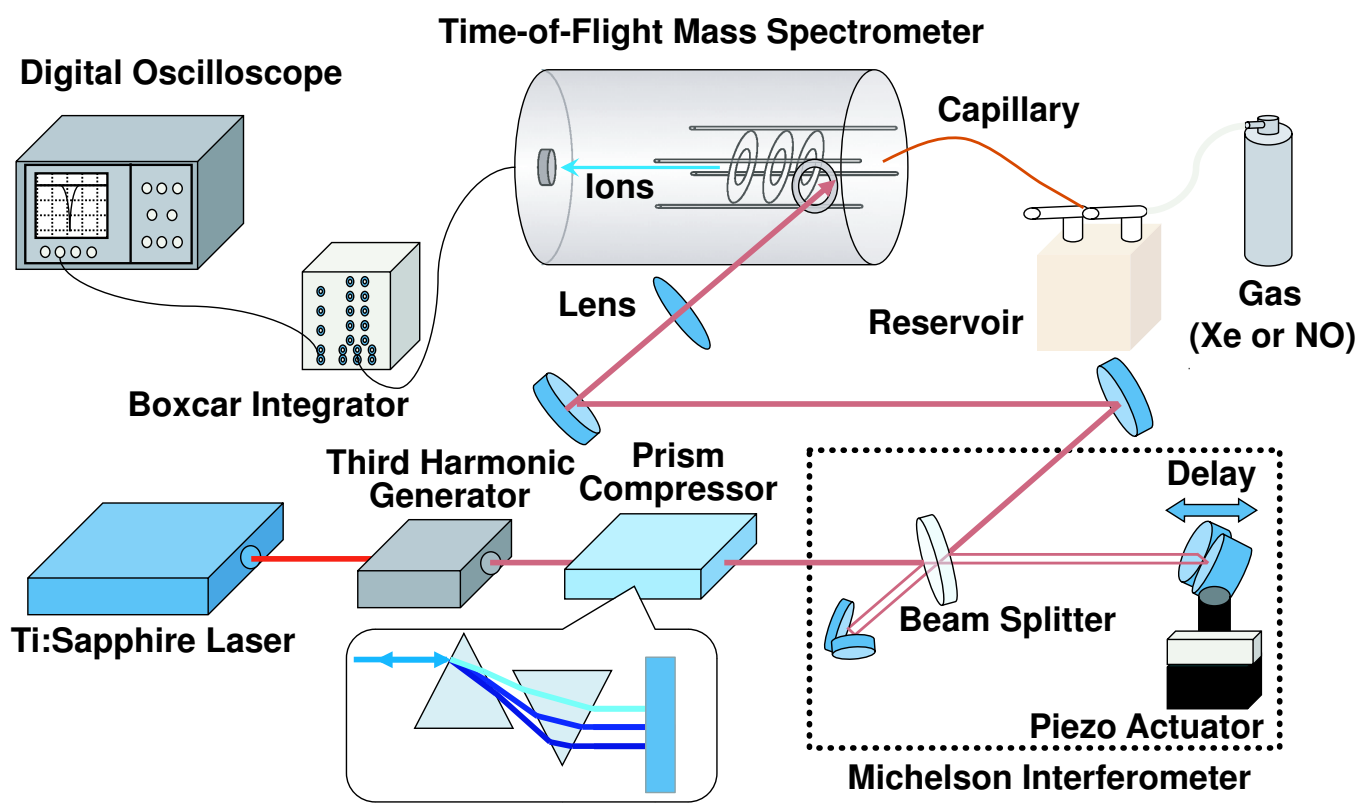

Fig. 2 Schematic diagram of the experimental setup. The reservoir was used for storing Xe and NO before they were injected into an MS. 


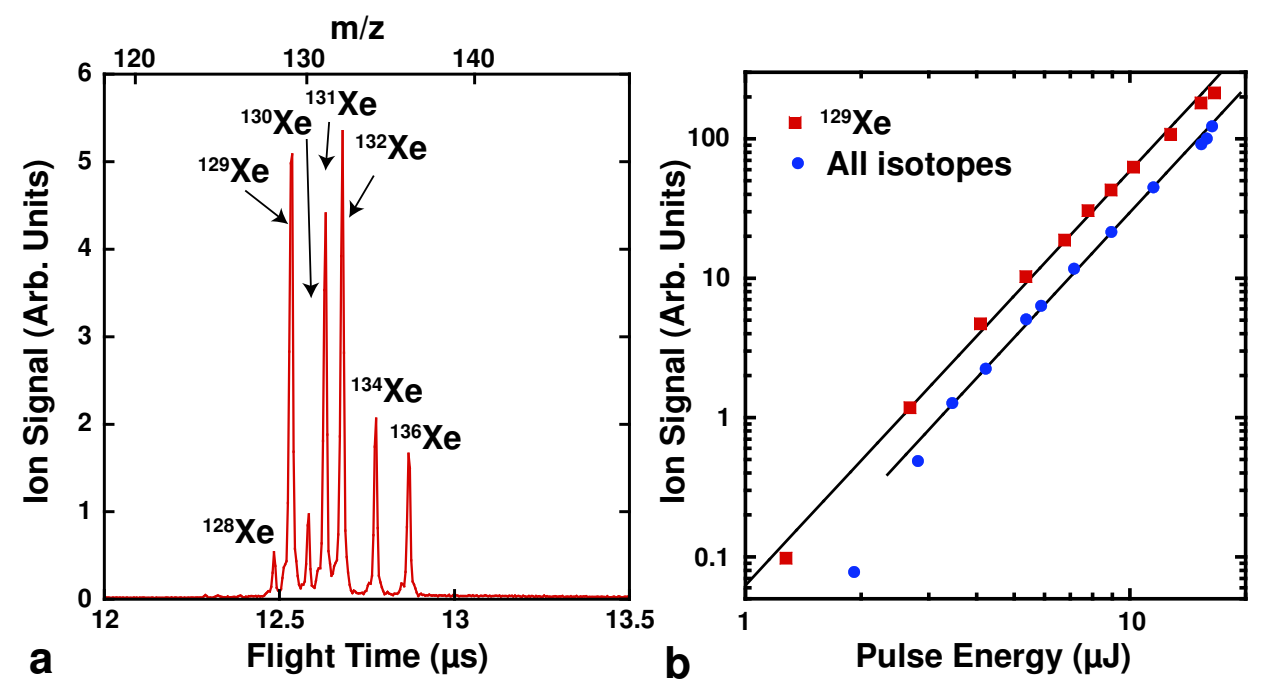

Fig. 3 (a) The time-of-flight mass spectrum for Xe isotopes measured using a DUV ultrashort optical pulse. (b) Dependence of the ion signals of ${ }^{129} \mathrm{Xe}$ (solid square) and all isotopes (solid circle) on the input pulse energy. 

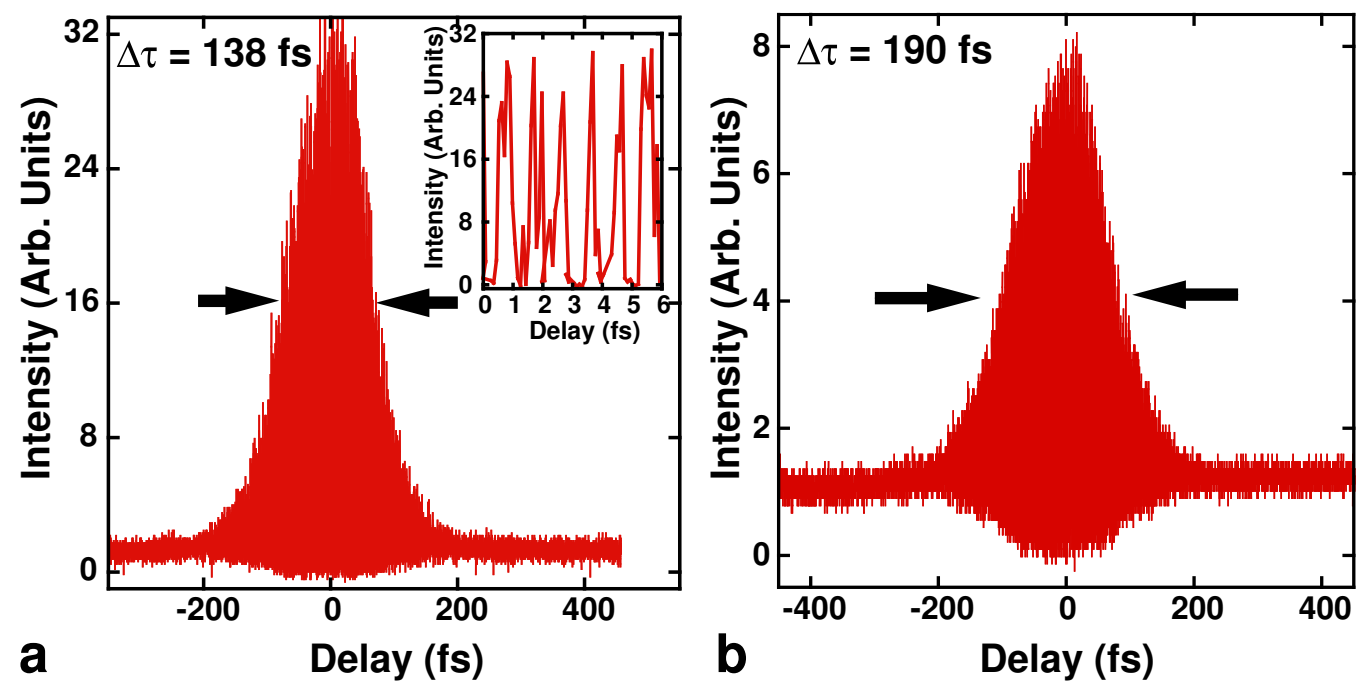

Fig. 4 The fringe-resolved autocorrelation traces of an FTL DUV pulse measured using the ion signal of (a) Xe isotopes and (b) NO. Interferometric fringe pattern is shown in the inset of (a). 

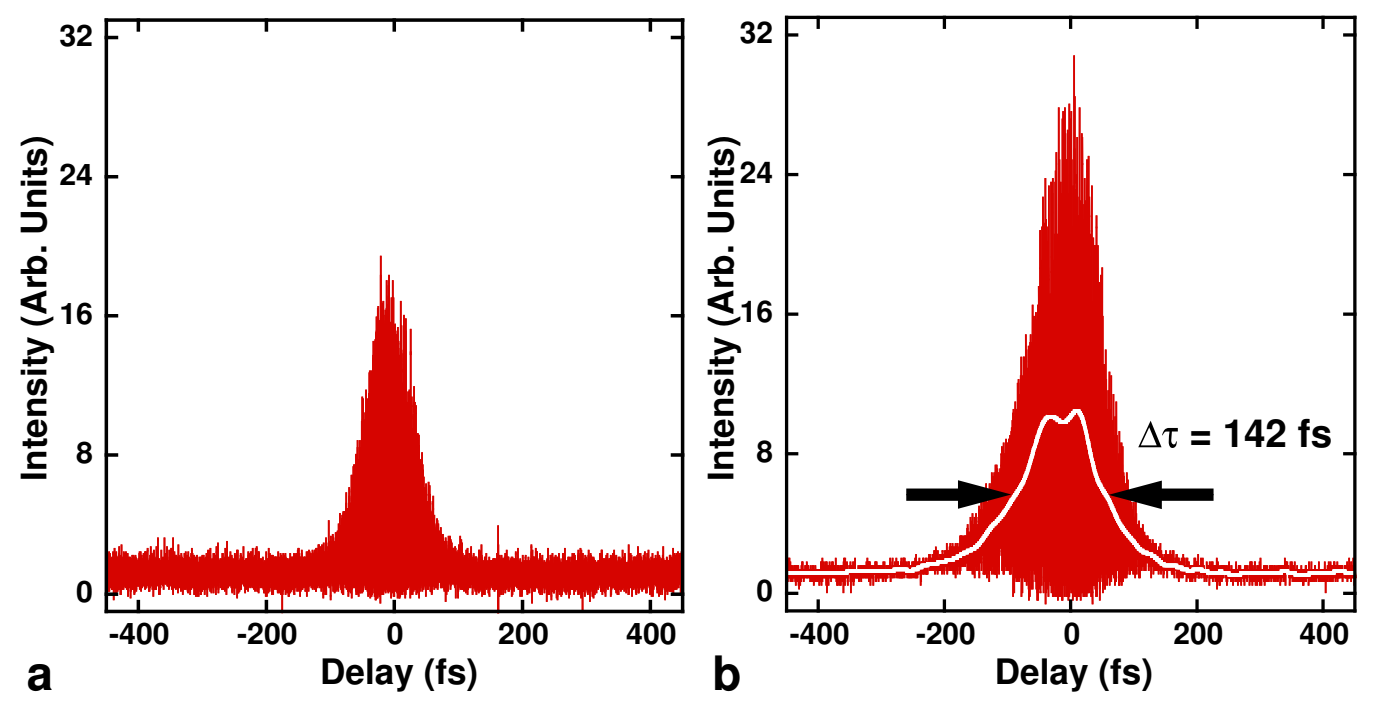

Fig. 5 The Xe-based FRAC traces of an FTL pulse at a pulse energy of $1.2 \mu \mathrm{J}$ and (b) a chirped pulse at a pulse energy of $15 \mu \mathrm{J}$. 
Table 1 The ratio of the FWHM in the autocorrelation trace to the pulse width in real time for typical (Gaussian and $\operatorname{sech}^{2}$ ) pulse shapes and $n=2$ or 3 signal nonlinearity.

\begin{tabular}{ccccc}
\hline & \multicolumn{2}{c}{ Gaussian } & \multicolumn{2}{c}{$\mathrm{sech}^{2}$} \\
\hline$n$ & $I_{\text {frac }}$ & $I_{\text {iac }}$ & $I_{\text {frac }}$ & $I_{\text {iac }}$ \\
\hline 2 & 1.696 & 1.414 & 1.897 & 1.543 \\
3 & 1.331 & 1.225 & 1.352 & 1.286 \\
\hline
\end{tabular}

